\title{
One standard of care for all is not always practical
}

\author{
ROBYNA IRSHAD KHAN
}

Clinical Lecturer and Consultant Anaesthetist, Department of Anaesthesia, Aga Khan University, Stadium Road, Karachi, PAKISTAN e-mail: robyna.khan@aku.edu

\begin{abstract}
Multiple research guidelines address the issue of standard of care in international collaborative research. These guidelines fail to appreciate that differing standards may be present within the same country, which makes their application sometimes impracticable.In circumstances where ethics review committees follow one of these guidelines entirely and to the hilt, some relevant and useful research is rejected while the way for "me too" drug trials is paved. It should be acceptable to hold different researchers to separate standards of care on the basis of their intentions, their financial resources, their ultimate gains from the research, and subsequent utilisation of the results of the research, even when these researchers come from the same country where the research is being conducted.
\end{abstract}

Key words: standard of care, research ethics

Should a universal standard of care be adhered to in all types of research conducted anywhere in the world? This is an excessively debated question in research ethics (1). Multiple international guidelines try to provide an ethical framework to prevent exploitation when financially robust sponsors from industrialised countries conduct research on vulnerable populations in resource-poor countries $(2,3,4)$. Unfortunately, these guidelines do not address the issue from the perspective of local researchers coming from varied backgrounds. Factors associated solely with the researchers like their intentions, their material resources, their ultimate gains from the research, and the subsequent utilisation of the results of the research must be given due consideration while deciding on the standard of care. If all these factors are considered diligently, it seems only reasonable to allow different standards of care within the same country in different settings; it is inappropriate to compel researchers to provide the best care available anywhere in the world, especially where they are not being supported by sponsors.

\section{Case 1}

Dr $\mathrm{K}$ is a family physician working in a basic health unit of a small rural community in Pakistan. During his clinical practice, he observes that children under the age of five are suffering from a typhoid fever that is resistant to the locally available drugs. He designs a small randomised control trial to determine whether a combination of more than one of these drugs is effective. His study design employs the available single-dose regimen for the control group. The best proven treatment for typhoid fever, intravenous third-generation antibiotics, is not available in his hospital and he has no resources to provide for them. The research ethics board rejects the proposal, as the best proven treatment is not being compared to the studied drug combination.

\section{Case 2}

A national pharmaceutical company in Pakistan has developed a newer type of insulin, Insulin-D, to treat Insulin Dependent Diabetes Mellitus (IDDM). This company intends to start a large scale phase-III trial in a local rural community with a high prevalence of IDDM. A renowned researcher from a nearby city is selected to conduct the trial. The study design employs the locally available standard of care for the control group. This is Insulin- $B$, that has lately been ineffective to manage blood sugar levels. The research ethics board asks the research team to modify the study design and provide the best proven therapy, Insulin-C, to the control group. The primary investigator changes the protocol accordingly and assures the availability of Insulin$C$ for both the control and study groups for one month after completion of this project. The sponsors declare a hefty budget for the overheads besides the trial expenses. They agree to market the study drug in the community (if it proves effective) but refuse to make it available free of cost for more than a month after the research is finished. In addition, they cannot guarantee that the drug, when marketed, will be available at a cost affordable to the community. However, they offer to compensate the community by building a clinic in the village. The proposal gets approved.

\section{Discussion}

On the surface, the decisions of the research ethics boards look genuine. In the first instance, the study protocol was not approved, as the researcher was not offering the best proven therapy for the control group though it is available in the country. The second was approved when the researcher agreed to use the best proven treatment. Let us analyse them in detail.

The intention of $\mathrm{Dr} \mathrm{K}$ in the first case is to cure a treatable disease when the causative organism has developed resistance to the available therapy. His goal is to provide the community with a desperately needed drug, without extra cost. He is using the patients as a means to an end, but not merely as means. The pharmaceutical company, on the other hand, is using these patients only as the means. Though the research participants need insulin, they will not have access to it after the conclusion of the trial. The company has no intention of providing the patients or their community with either Insulin-D or the best proven therapy, Insulin-C. This trial should preferably be 
conducted in a sub-group of private sector patients where the drug will be marketed in view of the fact that justice requires an equitable distribution of harms and benefits (5).

Dr $\mathrm{K}$ does not have the financial resources to provide for thirdgeneration antibiotics to the control group. As a result, his choices are limited to either using the best locally available treatment or giving up the idea altogether. If he is forced to give up this study, the best interests of the community are not being served. The pharmaceutical company, on the other hand, has declared a hefty overhead budget. Provision of the best proven treatment is merely a procedural issue for them. Researchers in non-industrialised countries commonly insist on providing the best locally available standard of care, arguing that it is not practicable to sustain the best proven treatment (6). Those conducting trials of "me too" drugs with huge marketing potentials should be held to the highest ethical standards with the proviso that they must sustain this standard for as long as the research participants require it.

There is no possibility of $\mathrm{Dr} \mathrm{K}$ making personal financial gains from the study he proposes. On the other hand, the pharmaceutical company is conducting this trial only for financial gain. It is willing to provide Insulin- $C$ to trial participants for a month after the study is completed, but do not feel responsible for their remaining life span.Since Insulin Dependent Diabetes Mellitus necessitates life-long use of insulin, these patients will have to revert to the ineffective Insulin-B after a month. The pharmaceutical company is making a clinic for the community. But there are many instances of such infrastructure ending up as a burden to the local community which is unable to meet exorbitant recurring expenses $(7,8)$. The fate of the building that is being offered is not likely to be different.

The trial by $\mathrm{Dr} \mathrm{K}$ has potential benefits for the participating community on a long-term basis. In contrast, the trial by the drug company has no long-term benefit for the local community. Researchers conducting non-beneficial research for participating communities tend to provide alternative, onetime compensations. Any dealings with the research subjects should be above and beyond these financial incentives. If a decision is made to accept these arrangements, each research subject should be clearly informed that he or she will be eligible for the new insulin free of cost only for a month after the trial; they will get a clinic for the community instead. These incentives may compensate for the lack of long-term availability of the new drug for the whole community. But they should not be seen as an alternative to a sustainable standard of care for research subjects with chronic diseases.

\section{Conclusion}

Conditions of uniform standards of care are emphasised in all international ethical guidelines. Application of this uniform standard is good for accountability but sometimes it is an obstacle to relevant, essential research. It may also facilitate "me too" drug trials. Ethics review committees should take these guidelines for what they are - only guidelines. Medicine is a dynamic, ever changing field. Generalisation and application of universal frameworks to all situations is neither practical nor in the best interest of patients. The two cases cited above may look similar on the surface and may have been conducted in the same community, but their implications for the participants are very different. It is only fair to respond to these differences in different ways.

\section{References:}

1. Angell M. Investigator's responsibilities for human subjects in developing countries. N Engl J Med 2000; 342: 967-969.

2. Council for International Organizations of Medical Sciences. Guideline 11: International ethical guidelines for biomedical research involving human subjects. 2002 [Cited 2004 Feb 2]. Available from: http://www. cioms.ch/frame_guideline_nov_2002.htm.

3. World Medical Association Declaration of Helsinki. Guideline 29: Ethical principles for medical research involving human subjects. 2000 [Cited 2004 Feb 2]. Available from: http://www.wma.net/e/policy/b3.htm.

4. Nuffield Council on Bioethics. The ethics of research related to healthcare in developing countries: The appropriate standard of care for control group in clinical trials. 2002 [Cited 2004 Feb 2]. Available from: http:// www.nuffieldbioethics.org/publications/developingcountries/ rep0000000893.asp.

5. Beauchamp TL, Childress JF. Principles of Biomedical Ethics. $5^{\text {th }}$ ed. New York: Oxford University Press; 2001.p 226.

6. Bhutta ZA. Why regulate? The need for national guidelines for ethical regulation of research.J Coll Physicians Surg Pak 2001; 9: 537-540.

7. Varatharajan, Thankappan R, Jayapalan S. Assessing the performance of primary health centers under decentralized government in Kerala, India. Health Policy Plan 2004; 19:41-51.

8. Bureau of Program and Policy Coordination: US Agency for International Development. USAID policy paper recurrent cost: Problems in less developed countries. 1982 [Online] [Cited 2004 April 3]. Available from: http://www. usaid.gov/policy/ads/200/rec_cost/recurcst.doc. 